\title{
Raman amplification and pulse dynamics in silicon photonic crystal waveguides
}

\author{
Victor M. Fernandez Laguna ${ }^{1,2}$ and Nicolae C. Panoiu ${ }^{1}$ \\ ${ }^{I}$ Department of Electronic and Electrical Engineering, University College London, London, WC1 E7JE, UK \\ ${ }^{2}$ Airbus Defence and Space, Gunnels Wood Rd, Stevenage, SG1 2AS, UK \\ victor.laguna.15@ucl.ac.uk and n.panoiu@ucl.ac.uk
}

\begin{abstract}
A rigorous mathematical model is developed to study the Raman effect in silicon photonic crystal waveguides. We show how enhanced signal amplification without pulse distortion is achieved by adequately tuning the pulse group velocities.
\end{abstract}

OCIS codes: $190.5650,290.5910,160.5298,130.5296,130.4310$.

\section{Introduction}

We present a rigorous and comprehensive theoretical model to study Raman amplification in silicon photonic crystal waveguides (Si-PhCWGs), which incorporates key effects pertaining to the pulse dynamics, including group-velocity (GV) dispersion (GVD), self-phase modulation (SPM), cross-phase modulation (XPM), and the effect of photogenerated free carriers (FCs). Indeed, whereas Raman scattering has been intensively investigated in PhC fibers [1,2] and also in silicon waveguides with uniform cross-section [3], a complete study of pulsed Raman interaction in SiPhCWGs with all linear and nonlinear optical effects included is not available yet. In particular, our model predicts that signal amplification via Raman interaction is largely dependent on the walk-off length between pulses, and that the proper choice of the GV of the pump and signal pulses leads to large, distortionless amplification of the signal.

Raman scattering is the result of the interaction between two pulses, namely pump and signal, whose spectral difference is close to the vibrational Raman frequency of the medium. At resonance, this effect can lead to strong phonon mediated signal amplification. Due to the strong optical field confinement in ultra-small cross-section of SiPhCWGs, dispersion engineering can be employed to control the strength of Raman scattering in these waveguides, justifying the importance of studying this phenomenon in a comprehensive way.
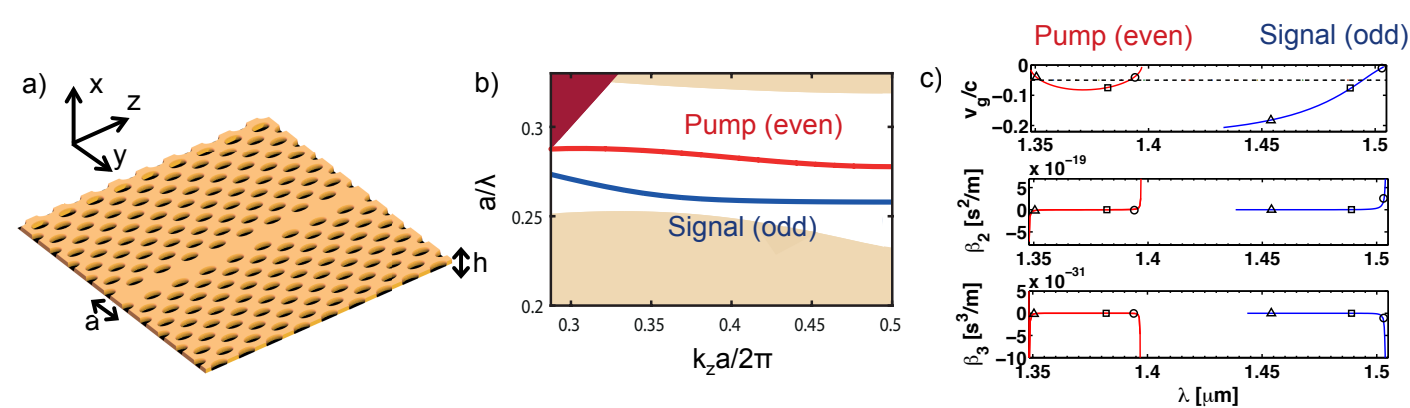

Fig. 1. (a) Schematics of the Si-PhCWG. (b) Projected band diagram of the waveguide. Red and blue curves represent the pump and signal guided modes, respectively. (c) Dispersion coefficients vs. wavelength for the pump and signal modes of a waveguide with $a=388 \mathrm{~nm}$.

\section{Silicon photonic crystal waveguide and mathematical model}

The Si-PhCWG structure considered in this study is commonly known as W1 PhCWG, and is made by removing a row in a honeycomb lattice of air holes in a homogeneous silicon slab, as per Fig. 1(a). The index of refraction of the silicon slab is $n_{S i}=3.48$, its height is $h=0.6 a$, and the radius of the holes is $r=0.29 a$, where $a$ is the lattice constant of the 
crystal. The projected bands corresponding to the guided modes of the waveguide are shown in Fig. 1(b), whereas the wavelength dependence of the dispersion coefficients of a waveguide with $a=388 \mathrm{~nm}$ are presented in Fig. 1(c).

The mathematical model we developed reduces to a set of coupled nonlinear Schrödinger (NLS) equations for the pump and signal, combined with a rate equation describing the carrier dynamics. In our model, the nonlinear coefficients corresponding to the SPM, XPM, and Raman interaction are calculated from the Kerr and Raman nonlinear susceptibilities using certain modal averages [3]. These nonlinear coefficients are inverse proportional to the square of the GV of the pump and signal pulses, as per Fig. 1(c) where the dashed line indicates the separation between slowand fast-light regimes. The circles, squares and triangles illustrate the three different scenarios analyzed in this paper: slow-slow, fast-fast, and slow-fast, respectively.

\section{Raman amplification versus pump and signal group velocities}

The coupled NLS equations are solved by the split-step Fourier method [4] for the three different scenarios mentioned above. When both the pump and the signal are in the slow-light region, Raman amplification coefficient becomes notably strong as $\gamma_{R}^{s} \propto\left(v_{g, p} \cdot v_{g, s}\right)^{-1}$; however, due to the walk-off length between pulses (around $50 \mu \mathrm{m}$ ) the amplification efficiency does not increase beyond the $-5 \mathrm{~dB}$ threshold. Maintaining the pump in the slow-light regime but shifting the signal to the fast-light regime can increase the walk-off length and boost the signal amplification up to $30 \mathrm{~dB}$, but, on the other hand, pulse distortion increases considerably due to enhanced SPM and XPM effects. Finally, when both pulses propagate in the fast-light regime, even though the Raman interaction is weaker, the walk-off length increases to the order of millimeters and distorsionless amplification of more than $20 \mathrm{~dB}$ is possible.

a)

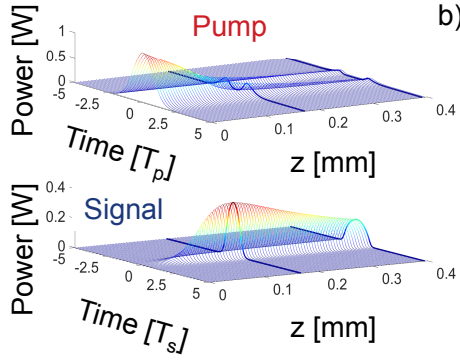

b)

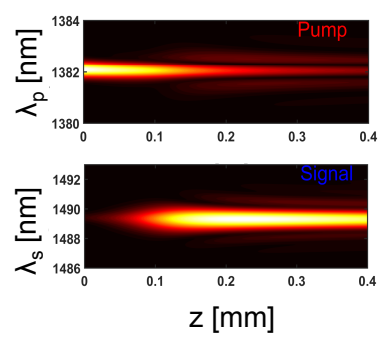

c)

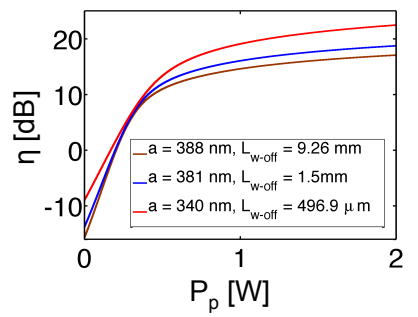

Fig. 2. (a) Pulse evolution in time domain for the fast-fast case: $a=388 \mathrm{~nm}, T_{0}=5 \mathrm{ps}$, and initial peak powers $P_{s}=5 \times 10^{-4} \mathrm{~W}$ and $P_{p}=1 \mathrm{~W}$. (b) Evolution of pump (top) and signal (bottom) spectra along the waveguide. (c) Raman amplification efficiency vs. pump peak power for different values of the walk-off length and for $T_{0}=10 \mathrm{ps}$ and $L_{w g}=1 \mathrm{~mm}$.

Figure 2(a) illustrates the pump depletion at the center of the pulse when the walk-off length is much bigger than the waveguide length, whereas Fig. 2(b) shows how SPM and XPM affect the pump and the signal, respectively. Indeed, SPM and XPM set in for pump peak powers above $\approx 0.7 \mathrm{~W}$. Finally, Fig. 2(c) reveals the smaller the lattice constant is, the earlier the pulses walk-off from each other and surprisingly more amplification is achieved, justified as not only the central region of the pump gets depleted but also one of the side regions, transferring more energy to the signal.

In conclusion, we demonstrated that the Raman interaction in silicon photonic crystal waveguides can lead to large signal amplification by adequately engineering the group velocities of the optical modes, and thus be a key enabler of active photonic nanodevices.

\section{References}

1. Benabid, F., Knight, J. C., Antonopoulos, G., et al., Stimulated Raman Scattering in Hydrogen-Filled HollowCore Photonic Crystal Fiber, Science, 298, pp. 399-402, 2002.

2. Coen, S., Chau, A.H.L., Leonhardt, R., et al., Supercontinuum generation by stimulated Raman scattering and parametric four-wave mixing in photonic crystal fibers, J. Opt. Soc. Am. B, 19, pp. 753-764, 2002.

3. X. Chen, N. C. Panoiu, and R. M. Osgood, Jr., Theory of Raman-Mediated Pulsed Amplification in Silicon-Wire Waveguides, IEEE J. Quantum Electron., 42, pp. 160-170, 2006.

4. Govind P. Agrawal, in Nonlinear Fiber Optics, 3rd edition, Academic Press, 2001. 